\title{
Promoção da saúde ocular na escola: percepções de professores sobre erros de refração
}

\author{
Ocular health promotion at school: the teacher's perception of refractive errors in the eye
}

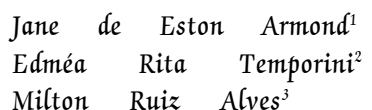

\section{RESUMO}

Objetivo: Identificar percepções de professores do sistema público de ensino, em relação aos erros de refração manifestados na idade escolar, a fim de subsidiar programas de treinamento para docentes, visando à detecção e posterior assistência a problemas oftálmicos de escolares. Métodos: Estudo transversal em população de professores de primeira série do ensino fundamental, de todas as escolas públicas da região sul do município de São Paulo. Aplicou-se questionário estruturado com base em estudo exploratório. Resultados: Foi obtida uma população de 545 sujeitos, distribuídos em 120 escolas. A população apresentou média de idade de 37,8 anos e média de tempo de magistério de 13,2 anos. A maioria (67,4\%) não recebeu orientação sobre saúde ocular nos últimos três anos. Os professores distinguiram mais corretamente os sinais de miopia (70,8\%) do que os de hipermetropia $(42,9 \%)$ e astigmatismo (40,9\%). Proporção significativa $(48,5 \%)$ apontou sinais e comportamentos indicativos da presença de miopia no escolar; $40,9 \%$ apontaram dificuldades na leitura e na escrita, para a criança hipermétrope sem correção óptica. Na criança astigmata as manifestações mais mencionadas foram "vista embaçada" $(45,4 \%)$ e desinteresse por atividades que exigem esforço visual (40,9\%). Os professores classificaram todos os erros de refração como agravos muito graves. Conclusão: Foram evidenciados conhecimentos distorcidos e/ou insuficientes entre professores do ensino fundamental, a respeito de erros de refração manifestados na idade escolar.

Descritores: Erros de refração/diagnóstico; Oftalmologia/educação; Acuidade visual; Saúde escolar; Promoção da saúde; Serviços de saúde escolar

INTRODUÇÃO

Múltiplas são as causas de perda da capacidade visual. Em geral, relacionam-se a fatores biológicos, sociais e ambientais, por vezes passíveis de serem evitados ou minimizados.

Especialistas reunidos pela "American Academy of Ophthalmology" para o estudo de causas de incapacidade visual e cegueira na América Latina, apontaram os erros de refração entre os distúrbios oculares mais freqüentes ${ }^{(1)}$.

Aproximadamente $15,0 \%$ das crianças em idade escolar necessitam uso de correção óptica, 4,0\% são amblíopes, $25,0 \%$ necessitam algum atendimento oftalmológico, sendo os erros de refração não corrigidos uma das principais causas de deficiência visual nas crianças no Brasil ${ }^{(2)}$.

Até a idade escolar, pode passar despercebida da família a maior parte das dificuldades visuais da criança, por desconhecimento e/ou ausência de sinais ou queixas. Ao ingresso na escola, contudo, manifestam-se distúr- 
bios oculares, pré-existentes ou não, evidenciados em razão do esforço visual necessário à realização do processo ensinoaprendizagem. Os problemas visuais influem no rendimento escolar e na sociabilização da criança, requerendo ações precoces de identificação e tratamento ${ }^{(3-4)}$.

O desenvolvimento de programas para detecção de distúrbios visuais de escolares do ensino fundamental, como forma de prevenção e correção de desvios da normalidade, requer a participação do professor. A convivência diária com os alunos propicia-lhe a oportunidade de conhecê-los e observar a ocorrência de alterações na aparência e no comportamento, provocadas por desvios da saúde ocular ${ }^{(3-5)}$.

Entre esses desvios da normalidade, salientam-se os erros de refração, considerando-se a freqüência com que ocorrem e a manifestação de sinais, sintomas e comportamentos passíveis de serem notados pelo professor ${ }^{(6)}$. Para isso, é necessário preparo específico do professor em saúde ocular, visando sua participação efetiva em programas de prevenção da incapacidade visual e da cegueira, na escola.

Realizou-se a presente pesquisa com o propósito de identificar percepções de professores do sistema público de ensino em relação aos erros de refração manifestados na idade escolar. A obtenção dessas informações teve a finalidade de subsidiar programas de treinamento de professores, a fim de contribuir para a detecção e assistência a problemas oftálmicos de escolares no sistema público de ensino.

\section{MÉTODOS}

Foi realizado um estudo transversal entre professores de primeira série do ensino fundamental, nas escolas do sistema público situadas na região sul do município de São Paulo (SPBrasil). A população foi composta pela totalidade desses professores, distribuídos em 120 unidades escolares.

Foram investigadas percepções relacionadas aos erros de refração e suas manifestações na idade escolar.

Realizou-se estudo exploratório que permitiu conhecer previamente a realidade e a terminologia empregada por professores em relação ao objeto da pesquisa. Observou-se que determinados termos técnicos de oftalmologia (miopia, astigmatismo, hipermetropia) eram conhecidos pelos professores. As informações foram obtidas por meio de entrevistas e reuniões com professores que apresentavam características semelhantes às da população de estudo. A partir dessas informações, foi construído um questionário estruturado, contendo perguntas e respostas ${ }^{(7)}$.

Optou-se por construir um questionário auto-aplicável, considerando critérios de viabilidade, acesso e características da população-alvo. $\mathrm{O}$ instrumento foi submetido a teste não recebeu críticas ou sugestões, sendo considerado o instrumento definitivo da pesquisa.

A coleta de dados foi realizada com o auxílio dos diretores das unidades de ensino, solicitado em reunião preparatória de sensibilização sobre a importância da pesquisa. Anexou-se carta introdutória a cada instrumento, informando sobre os objetivos da pesquisa e solicitando o preenchimento individual; foram garantidos o sigilo e anonimato das informações. Coube ao diretor da cada unidade de ensino, a responsabilidade pela distribuição e recolhimento dos questionários preenchidos. Os dados foram coletados em setembro de 1997 e processados utilizando-se o programa "Microsoft Excel".

A seleção de sinais indicativos dos erros de refração, baseou-se no conteúdo de manuais usados para treinamento sobre saúde ocular, no sistema público de ensino ${ }^{(8)}$.

Por se tratar de questionário auto-aplicado, o " $\mathrm{n}$ " das tabelas sofre algumas variações em função das respostas fornecidas.

\section{RESULTADOS}

A população foi composta por 545 professores de idades entre 20 e 69 anos, média de 37,8 anos, exercendo funções de magistério em média há 13,2 anos. A maioria $(67,4 \%)$ não havia recebido qualquer orientação sobre o sistema visual ou treinamento sobre saúde ocular na escola, nos últimos três anos.

As figuras 1 a 3 registram as percepções dos professores sobre sinais e sintomas indicativos dos erros de refração, que apresentam conseqüências visuais. Observa-se que os respondentes distinguem mais corretamente os sinais de miopia $(70,8 \%)$, do que os sinais de hipermetropia $(42,9 \%)$ e de astigmatismo $(40,9 \%)$. Nota-se uma porcentagem significativa das respostas "não sei", para ambos os casos: hipermetropia (31,3\%), astigmatismo (28,6\%).

Proporção significativa de respondentes apontou sinais e comportamentos indicativos da presença de miopia na criança $(24,7 \%$ e $23,8 \%)$, ou seja, $48,5 \%$ (Tabela 1 ).

A maioria dos entrevistados respondeu que a criança míope levanta-se da carteira $(69,0 \%)$ ou "aperta" os olhos $(68,5 \%)$, para enxergar melhor o que está escrito no quadro negro. Uma menor parcela dos respondentes $(41,0 \%)$ declarou que o escolar portador de miopia apresenta indisciplina; apenas $4,1 \%$ dos docentes têm a percepção que a criança míope prefere atividades de leitura (Tabela 1).

Quanto aos sinais e alterações de comportamento da criança hipermétrope sem correção óptica, registram-se proporções

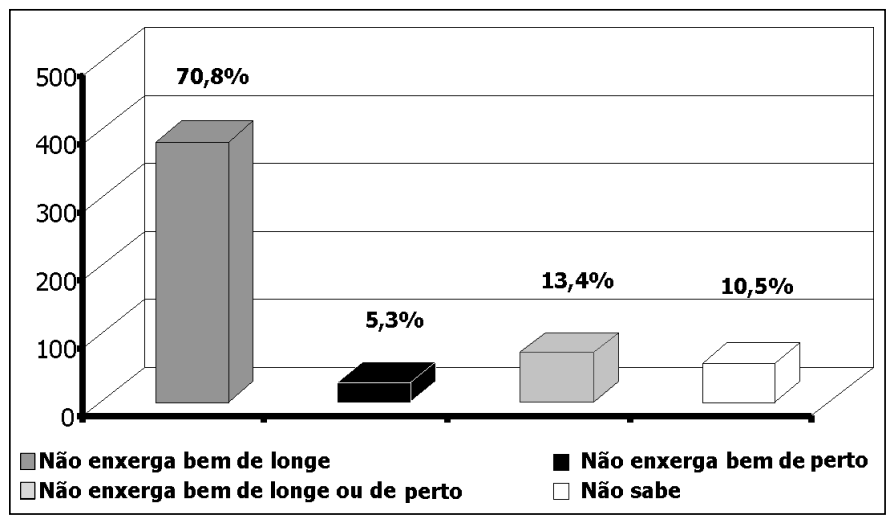

Figura 1 - Conhecimento de professores em relação a sinal indicativo de miopia em escolar. São Paulo, 1997 


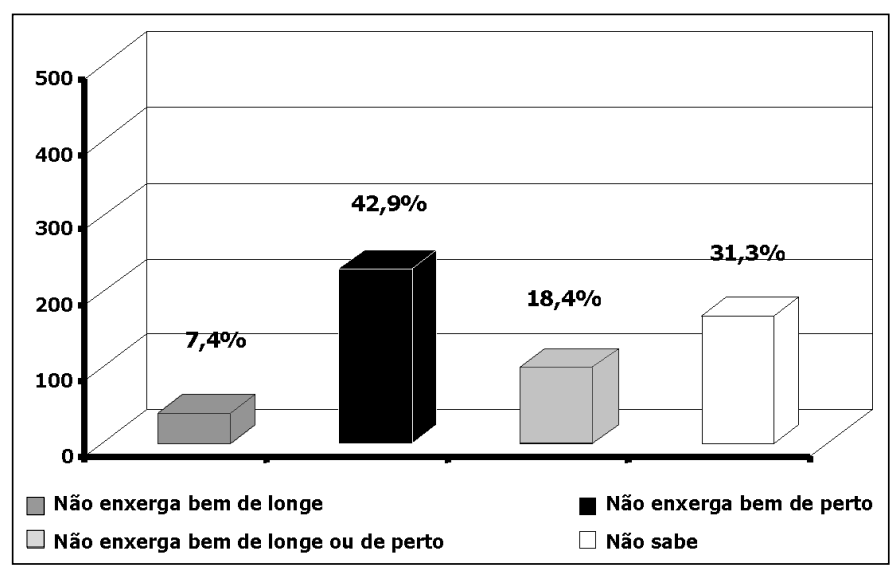

Figura 2 - Conhecimento de professores em relação a sinal indicativo de hipermetropia em escolar. São Paulo, 1997

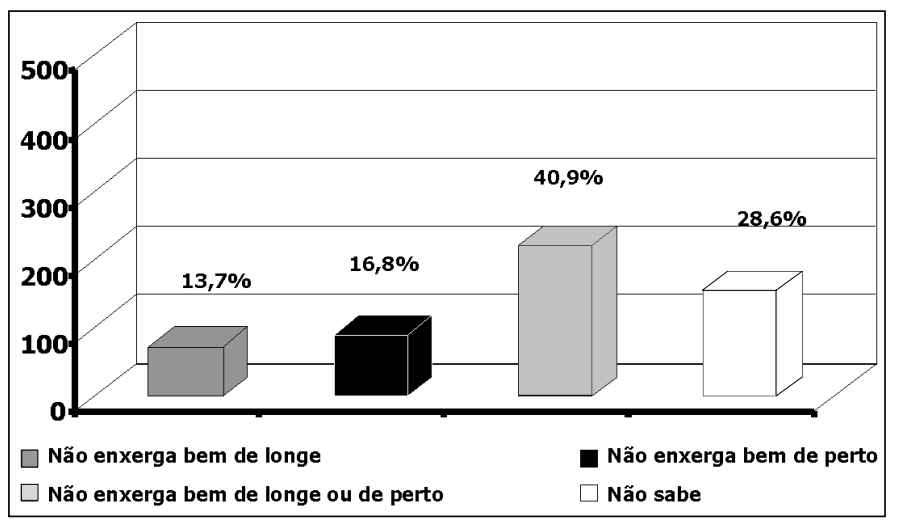

Figura 3 - Conhecimento de professores em relação a sinal indicativo de astigmatismo em escolar. São Paulo, 1997

reduzidas de respondentes na escolha das manifestações indicadas na tabela 2; por exemplo, apenas 7,6\% mencionam todas as respostas oferecidas. A maior parcela dos entrevistados (40,9\%), apontou dificuldades na leitura e na escrita, além de: dores de cabeça $(31,1 \%)$, olhos vermelhos pós esforço visual $(22,8 \%)$, cansaço pós leitura $(18,1 \%)$, tontura e enjôos $(17,7 \%)$ quando o escolar apresenta hipermetropia. Apenas $7,6 \%$ dos entrevistados responderam que o escolar hipermétrope tem preferência por esportes.

No que se refere ao astigmatismo, apenas $16,3 \%$ dos professores mencionam todas as manifestações apresentadas na tabela 3. O sinal menos reconhecido pelos respondentes consiste na presença de "olhos vermelhos" (19,6\%). Entre os mais apontados destacam-se apresentação de "vista embaçada" $(45,4 \%)$ e o desinteresse por atividades que exigem esforço visual $(40,9 \%)$, além de dores de cabeça $(33,0 \%)$ e esfregar os olhos $(26,5 \%)$.

Os resultados das tabelas 1, 2, e 3 evidenciam que os docentes têm conhecimento de alguns "sinais de alerta" dos erros de refração. Ressalte-se, entretanto, a proporção deles que declararam não saber quais seriam os sinais indicativos de miopia $(10,0 \%)$, hipermetropia $(28,0 \%)$, e astigmatismo $(22,8 \%)$.
Quanto à percepção sobre o grau de gravidade dos erros de refração, observa-se que a maioria considera todos os problemas oculares mencionados muito graves, com exceção de conjuntivites (Tabela 4).

\section{DISCUSSÃO}

A promoção da saúde ocular apresenta o princípio básico de melhoria da qualidade de vida do indivíduo, mediante a obtenção da capacidade visual que lhe permita o desenvolvimento de suas potencialidades e participação na sociedade ${ }^{(9)}$. A saúde e a qualidade de vida dependem do inter-relacionamento de múltiplos fatores psicossocioculturais e do ambiente, que possibilitam ou reforçam comportamentos do indivíduo $^{(10)}$. O conhecimento em relação à saúde ocular constitui um desses fatores, influindo na importância que as pessoas atribuem à visão e aos cuidados para preservá-la ${ }^{(3)}$.

O professor detém a tarefa precípua de transmissão de conhecimentos na escola, que pode incluir a orientação em saúde ocular para alunos e familiares. Para a realização dessa atividade, é necessário preparo específico que permita aos docentes a aquisição de conhecimentos, desprovida de distorções, provenientes de alguns mitos muitas vezes aceitos como verdades ${ }^{(11)}$. Entretanto, entre os professores que integraram o presente estudo a maioria declarou não ter sido orientada ou ter recebido qualquer tipo de treinamento sobre saúde ocular nos últimos três anos.

As ametropias podem provocar sintomas de astenopia e/ou comportamentos, passíveis de serem observados na escola pelos professores; essas alterações, conseqüentes da condição visual, muitas vezes não são percebidas pelos familiares ${ }^{(12)}$.

A hipermetropia, devido ao esforço acomodativo, pode causar cefaléia, hiperemia conjuntival. Tanto os sintomas pela presença de astenopia, como a deficiente acuidade visual nos

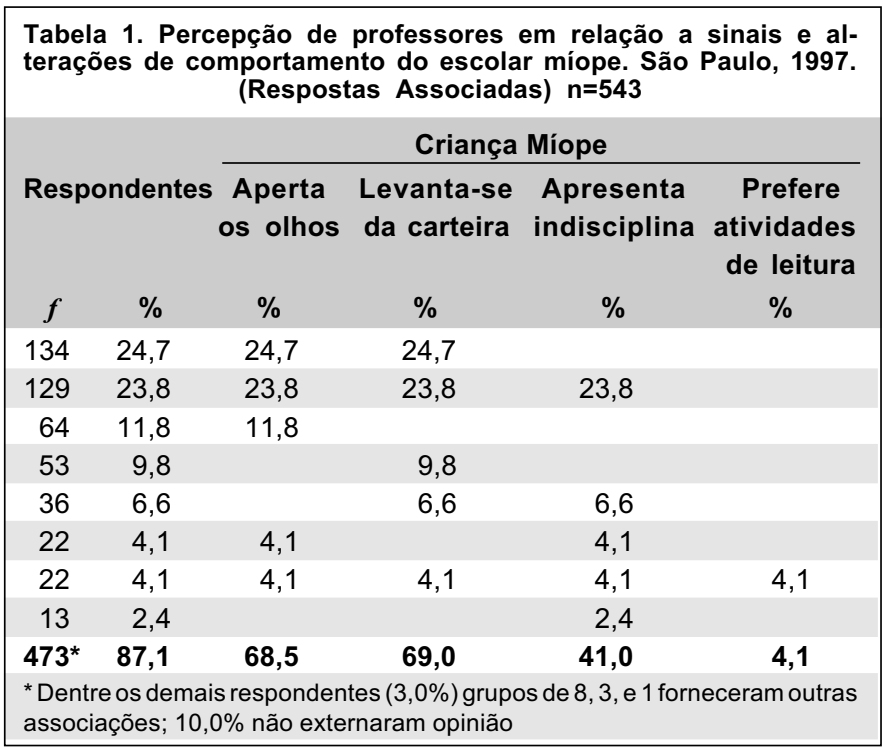




\begin{tabular}{|c|c|c|c|c|c|c|}
\hline \multirow{2}{*}{\multicolumn{2}{|c|}{ Respondentes }} & \multirow{3}{*}{$\begin{array}{c}\text { Dificuldade na } \\
\text { leitura e escrita } \\
\%\end{array}$} & \multicolumn{4}{|c|}{ Criança hipermétrope } \\
\hline & & & \multirow{2}{*}{$\begin{array}{l}\text { Dores de } \\
\text { cabeça } \\
\%\end{array}$} & \multirow{2}{*}{$\begin{array}{c}\text { Tontura e } \\
\text { enjôos } \\
\%\end{array}$} & \multirow{2}{*}{$\begin{array}{c}\text { Olhos vermelhos } \\
\text { pós-esforço visual } \\
\%\end{array}$} & \multirow{2}{*}{$\begin{array}{c}\text { Cansaço } \\
\text { pós-leitura } \\
\%\end{array}$} \\
\hline$f$ & $\%$ & & & & & \\
\hline 41 & 7,6 & 7,6 & 7,6 & 7,6 & 7,6 & 7,6 \\
\hline 31 & 5,8 & 5,8 & 5,8 & & & \\
\hline 25 & 4,6 & 4,6 & 4,6 & & 4,6 & \\
\hline 24 & 4,5 & 4,5 & 4,5 & 4,5 & 4,5 & 4,5 \\
\hline 19 & 3,5 & 3,5 & & & 3,5 & \\
\hline 18 & 3,3 & 3,3 & & & & \\
\hline 16 & 3,0 & 3,0 & 3,0 & 3,0 & & \\
\hline 16 & 3,0 & 3,0 & & & & 3,0 \\
\hline 16 & 3,0 & 3,0 & 3,0 & & & 3,0 \\
\hline 14 & 2,6 & 2,6 & 2,6 & 2,6 & 2,6 & \\
\hline $220^{*}$ & 40,8 & 40,9 & 31,1 & 17,7 & 22,8 & 18,1 \\
\hline
\end{tabular}

\begin{tabular}{|c|c|c|c|c|c|c|}
\hline \multirow{2}{*}{\multicolumn{2}{|c|}{ Respondentes }} & \multirow{3}{*}{$\begin{array}{c}\text { Desinteresses por } \\
\text { atividades com } \\
\text { esforço visual } \\
\%\end{array}$} & \multicolumn{4}{|c|}{ Criança astigmata } \\
\hline & & & Dores de & Olhos & Esfrega & Vista \\
\hline$f$ & $\%$ & & $\%$ & $\%$ & $\%$ & $\%$ \\
\hline 88 & 16,3 & 16,3 & 16,3 & 16,3 & 16,3 & 16,3 \\
\hline 29 & 5,4 & 5,4 & & & & 5,4 \\
\hline 28 & 5,2 & & 5,2 & & & 5,2 \\
\hline 25 & 4,6 & 4,6 & & & & \\
\hline 24 & 4,4 & & & & & 4,4 \\
\hline 23 & 4,3 & 4,3 & 4,3 & & 4,3 & 4,3 \\
\hline 21 & 3,9 & 3,9 & 3,9 & & & 3,9 \\
\hline 18 & 3,3 & 3,3 & 3,3 & 3,3 & & \\
\hline 17 & 3,1 & 3,1 & & & 3,1 & 3,1 \\
\hline 15 & 2,8 & & & & 2,8 & 2,8 \\
\hline $288^{*}$ & 53,3 & 40,9 & 33,0 & 19,6 & 26,5 & 45,4 \\
\hline
\end{tabular}

altos graus de hipermetropia, podem tornar a criança desinteressada pela leitura e por trabalhos manuais ${ }^{(2)}$.

Os sintomas de astenopia são mais acentuados no astigmatismo de pequeno ou médio porte, observando-se acuidade visual sensivelmente baixa nos altos graus dessa ametropia $^{(2)}$.

Quando os graus de miopia apresentam-se maiores do que -0,50 dioptrias, não corrigidas a criança limita as atividades que exijam visão à distância preferindo a leitura ou atividades que permitam trazer o objeto próximo aos olhos. Para melhorar a acuidade visual "aperta os olhos", levanta-se da carteira para copiar a lição do quadro negro, pode tornar-se inquieta ou tímida ${ }^{(2)}$. A razão da preferência da criança míope por atividades de leitura, ser o sinal menos conhecido pelos professores, deve-se provavelmente ao fato de ser a leitura, atividade pouco praticada hoje em dia, inclusive em sala de aula.
Os erros de refração foram considerados problemas oftálmicos graves pela maioria dos respondentes, porém ainda proporção significativa os considera de pouca ou nenhuma gravidade ou não souberam referir. Os erros de refração foram referidos como problemas quase tão graves quanto a ambliopia e o estrabismo, fato que evidencia desconhecimento em relação a esses problemas oculares. Isto reflete a percepção dos professores em relação às conseqüências negativas que a não identificação de erros de refração e a não prescrição da correspondente correção óptica podem trazer para o desenvolvimento do escolar. A ambliopia e o estrabismo podem resultar de erros refracionais não detectados e não tratados precocemente.

A conjuntivite e o tracoma constituem doenças infecciosas. A conjuntivite é a doença infecciosa ocular mais comum, auto-limitada, que evolui para a cura sem seqüelas na maioria dos casos. O tracoma foi considerado o mais grave distúrbio, 


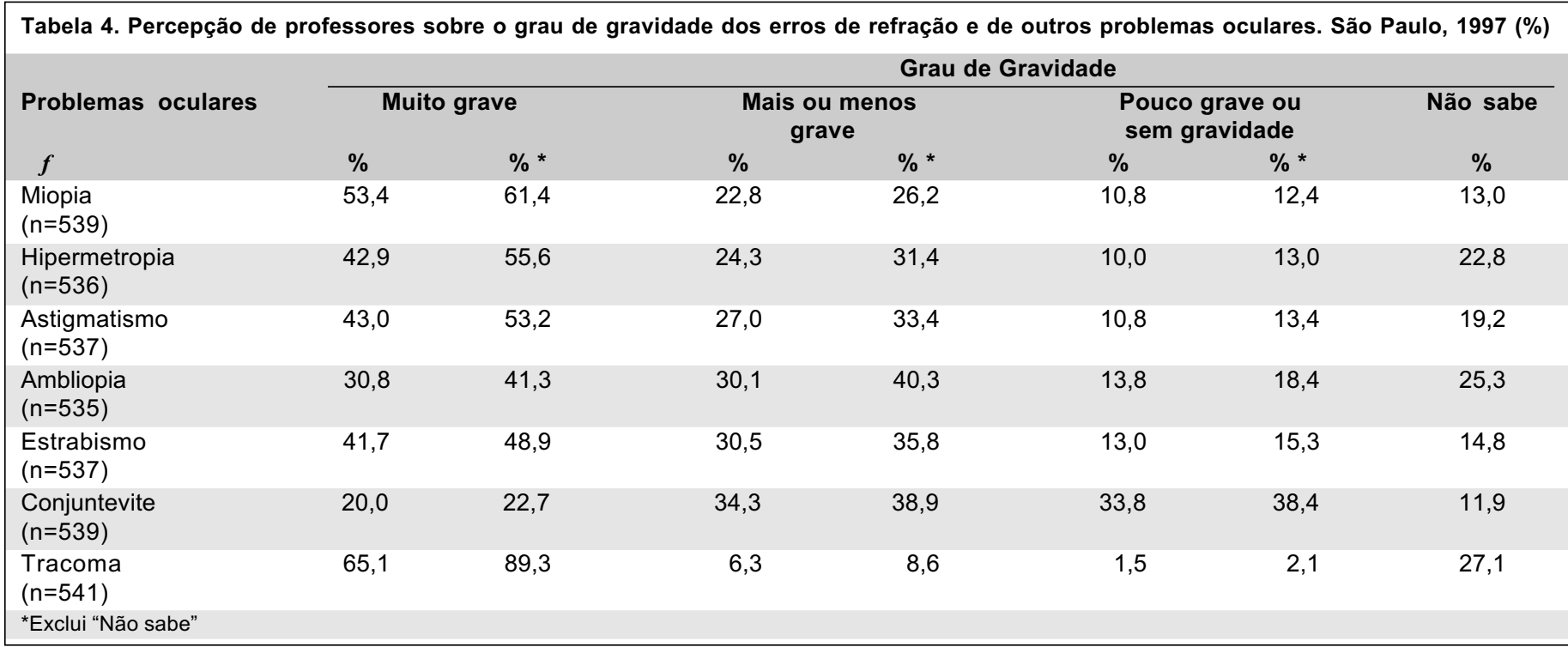

talvez por ser doença de notificação compulsória e demandar sempre uma ação imediata da equipe de saúde da região, na busca ativa de casos.

$O$ fato de que os representantes consideraram erro de refração como um agravo importante, de repercussão visual, provavelmente representa o efeito de alguma intervenção educativa na escola por programas de oftalmologia sanitária, ou de atenção à saúde do escolar.

Convém ressaltar, contudo, que a percepção da gravidade de uma doença baseia-se não apenas no conhecimento científico disponível, mas também em concepções populares de origem cultural e na interpretação de experiências vivencia$\operatorname{das}^{(11)}$.

Tendo em vista os resultados do presente estudo e considerando-se a importância do papel do professor na identificação do aluno com problemas de visão, faz-se necessária a orientação dos docentes, visando ampliar seu conhecimento e compreensão a respeito do papel fundamental que desempenham nas ações de saúde ocular na escola.

Sugere-se a implantação e efetivação de Programa de Saúde Ocular em todo o sistema público de ensino, visando desenvolver ações de prevenção da incapacidade visual e da cegueira, promoção e recuperação da saúde ocular.

\section{A B S T RAC T}

Purpose: Identification of the perception that teachers belonging to the public education system have concerning the refractive errors in the eye occurring at school age in order to subside training programs for teachers aiming at the detection of, and subsequent assistance to, ophthalmic problems in schoolchildren. Methods: A transversal study carried out with a population of first-degree elementary school teachers, covering all public schools of the southern region of
São Paulo, Brazil. A structured questionnaire based on an exploratory story was handed out. Results: The population comprised 545 subjects distributed among 120 elementary schools. The average age of the population was 37.8 years, with an average length of teaching time of 13.2 years. The majority of them $(67.4 \%)$ did not receive orientation on ocular health in the last three years. The teachers distinguished more accurately the symptoms of myopia (70.8\%) than those of hyperopia (42.9\%) and astigmatism (40.9\%). A significant number $(48.5 \%)$ of them pointed out signals and behaviors indicative of the presence myopia in schoolchildren; $40.9 \%$ indicated difficulties in reading and writing in hyperope children without optical correction. "Blurred vision" (45.4\%) and disinterest for activities demanding visual effort (40.9\%) were the most indicated manifestations in astigmatic children. All refractive errors in the eye were classified as very serious disorders by the teachers. Conclusion: Distorted and/or insufficient knowledge was disclosed among elementary school teachers, about refractive errors occuring at school age.

Keywords: Refractive errors/diagnosis; Ophthalmology/ education; Visual acuity; School health; Health promotion; School health services

\section{REFERENCIAS}

1. Verdaguer TJ. One world, one voice, one vision. Arq Bras Oftalmol 1998; 61:5-6.

2. Kara-José N, Alves MR. Problemas oftalmológicos mais freqüentes em escolares. In: Conceição JAN. coordenadora. Saúde escolar: a criança, a vida e a escola. São Paulo: Sarvier; 1994. p. 195-203.

3. Temporini ER. Prevenção de problemas visuais de escolares: conduta de professores do sistema de ensino do Estado de São Paulo, Brasil. Rev Bras Saúde Esc 1990;1:68-75. 
4. Conselho Brasileiro de Oftalmologia. Campanha Veja Bem Brasil - 1998: manual de orientação. São Paulo: Imprensa Oficial do Estado; 1998.

5. Kara-José N, Temporini ER. Avaliação dos critérios de triagem visual de escolares de $1^{a}$ série do $1^{\circ}$ grau. Rev Saúde Pública 1980;14:205-14.

6. Alves MR, Kara-José N. O olho e a visão: o que fazer pela saúde ocular de nossas crianças. Petrópolis: Vozes; 1996. 151 p.

7. Piovesan A, Temporini ER. Pesquisa exploratória: procedimento metodológico para o estudo de fatores humanos no campo da saúde pública. Rev Saúde Pública 1995;29:318-25.

8. São Paulo (Estado). Comissão Conjunta da Secretaria da Saúde e da Educa- ção. Plano de Oftalmologia Sanitária Escolar. São Paulo: Serviço de Oftalmologia Sanitária do Instituto de Saúde; 1976.

9. Temporini ER. Promoção da saúde ocular. Arq Bras Oftalmol 1999;62:82-4.

10. Briceño-Leon R. Siete tesis sobre la educacion sanitária para la participacion comunitária. Cad Saúde Pública 1996;12:7-30.

11. Armond JE, Temporini ER. Crenças sobre saúde ocular entre professores do sistema público de ensino no município de São Paulo - SP, Brasil. Rev Saúde Pública 2000;34:9-14.

12. Temporini ER. Percepção de professores do Estado de São Paulo, sobre seu preparo em saúde do escolar. Rev Saúde Pública 1988;22:411-21.

\section{Conselho Brasileiro de Oftalmologia}

\section{Provas do Concilium Universale}

Apresentamos as informações sobre as Provas do Concilium Ophthalmologicum Universale de 2002: International Basic Science Assessment Test (parte I e II) e International Clinical Science Assessment, realizadas simultaneamente em vários países, inclusive no Brasil, e em diferentes idiomas, que avaliam o nível de conhecimento dos Oftalmologistas.

Ressaltamos que um certificado de capacitação profissional expedido pelo "International Council of Ophthalmology" é extremamente valorizado pelas instituições estrangeiras e possibilita aos aprovados condições para obtenção dos melhores estágios no exterior.

O Basic Science Assessment Test é composto de duas partes: parte I - 80 questões sobre Ciências Básicas em Oftalmologia; parte II - 20 questões sobre óptica e refração. O teste todo tem duração de três horas. Médicos que já foram aprovados na parte I e reprovados na II, podem repetir esta última.

Clinical Science Assessment, destinado apenas aos aprovados no Basic Science Assessment, é composto de 200 questões com duração de quatro horas.

Podem se inscrever, médicos com Título de Especialista em Oftalmologia ou matriculado em Curso de Especialização em Oftalmologia.

Os interessados em se inscrever, devem entrar em contato até 14 de novembro próximo com a Srta. Iza Castro - Dept ${ }^{\circ}$ de Ensino, pelo telefone: (0xx11) 3266-4000 ou pelo e-mail oftalmo@cbo.com.br e solicitar o formulário de inscrição.

\section{TAXA DE INSCRIÇÃO}

Basic Science Assessment - parte I e II $\mathrm{R} \$ 323,00$

Basic Science Assessment - parte II R\$ 163,00 Clinical Science Assessment $R \$ 456,00$

A prova acontecerá no dia 14 de março de 2002. Juntamente com os formulários, os inscritos receberão as informações necessárias. 